\title{
Effect of Sildenafil in an Experimental Model of Ischemic Colitis
}

\author{
Bikash Medhi $\cdot$ Harjot Kaur $\cdot$ Ajay Prakash
}

Published online: 31 October 2008

(C) Springer Science+Business Media, LLC 2008

\section{Dear Sir,}

Irkorucu et al. [1] have provided interesting information regarding the potential benefits of sildenafil on an animal model of ischemic colitis. Their study showed very interesting results with sildenafil with a probable suggested mechanism as it enhances vasodilatation by relaxing the smooth muscle in the vessels and inhibits platelet aggregation. Therefore, this drug may be a valuable therapeutic target for ischemic colitis in the near future. We would like to highlight some of the points regarding this study:

1. All animals were sacrificed following $72 \mathrm{~h}$ after devascularization. The devascularization was done by cautery, which results in permanent damage to blood vessels, so how is vasodilatation going to help once the vessels are permanently damaged. Besides, this study did not show any development of collaterals in the histological findings.

2. There is no positive control group included in the study, since other vasodilators, like papaverine, are preferred in the treatment of ischemic colitis [2].

3. Besides this, other established markers should have been assessed, such as preliminary major cytokines for colitis, including TNF- $\alpha$, IL-1 $\beta$, IL-6, IL-12, and IL-23 [3].
The study provides useful information for more effective and better targeted treatment for patients with ischemic colitis, as the results showed the efficacy of sildenafil with probable enhanced vasodilatation by relaxing the smooth muscle and enhancing microcirculatory hemodynamics. However, the above mentioned points should be discussed for future therapeutic implication and reconfirmation in clinical studies.

\section{References}

1. Irkorucu O, Taşcilar O, Cakmak GK, et al. The effect of sildenafil on an animal model for ischemic colitis. Dig Dis Sci. 2008;53(6): 1618-1623. doi:10.1007/s10620-007-0033-9.

2. Herskowitz MM, Gillego V, Ward M, Wright G. Cocaine-induced mesenteric ischemia: treatment with intra-arterial papaverine. Emerg Radiol. 2002;9(3):172-174.

3. Hwang RF, Schwartz RW. Ischemic colitis: a brief review. Curr Surg. 2001;58(2):192-194. doi:10.1016/S0149-7944(00)00468-2.

B. Medhi $(\bowtie) \cdot$ H. Kaur · A. Prakash 